Service social

\title{
Récents développements et orientations actuelles de la théorie du service social en Italie
}

\section{Elisabetta Neve}

Volume 42, numéro 3, 1993

Perspectives théoriques

URI : https://id.erudit.org/iderudit/706632ar

DOI : https://doi.org/10.7202/706632ar

Aller au sommaire du numéro

Éditeur(s)

École de service social de l'Université Laval

ISSN

1708-1734 (numérique)

Découvrir la revue

Citer cet article

Neve, E. (1993). Récents développements et orientations actuelles de la théorie du service social en Italie. Service social, 42(3), 83-101.

https://doi.org/10.7202/706632ar
Résumé de l'article

L'article propose une synthèse de quelques acquis théoriques récents du service social italien en lien avec le contexte de pratique et l'évolution globale des politiques sociales des dernières années. L'auteure a choisi de concentrer son analyse sur la redéfinition des objectifs et des critères opérationnels généraux de la profession plutôt que sur des questions techniques et méthodologiques. Le fait que l'identité professionnelle du service social et les nouveaux contextes dans lesquels et avec lesquels il travaille s'influencent réciproquement justifie de privilégier le thème suivant: l'organisation institutionnelle des services et de la communauté territoriale locale en tant que sujets protagonistes potentiels des interventions en réponse aux problèmes sociaux. 


\title{
CONTRIBUTIONS \\ INTERNATIONALES
}

\section{Récents développements et orientations actuelles de la théorie du service social en Italie*}

\author{
Elisabetta NEVE \\ Professeure \\ École de service social \\ Université de Venise
}

À l'heure actuelle, la plupart des travailleuses et travailleurs sociaux en Italie exercent leurs fonctions à l'intérieur d'organismes du gouvernement local et leurs compétences concernent, dans l'ensemble, les interventions sociales d'assistance sanitaire destinées à la communauté territoriale.

Cela nous semble être une caractéristique de notre pays, attribuable aux transformations culturelles, politiques et institutionnelles des années 70 et 80 , qui ont comporté d'intéressants changements dans le travail pratique et théorique du service social. Sous la poussée d'une exigence

* Le plan de cet article a été élaboré avec la précieuse collaboration de Silvana Tonon Giraldo, professeure à l'École de service social de l'Université de Venise et membre de l'Association italienne des professeurs et professeures de service social. Tout aussi précieuses ont été ses suggestions à la phase de révision du texte. L'auteure, tout en exprimant sa reconnaissance à Silvana Giraldo, remercie aussi Giuseppina Gottardi, professeure à l'École de service social de la même université, pour ses conseils pendant la rédaction de l'article, de même que Sonia Bourquenez-Branca pour la traduction du texte en français. NDLR - Gérald Doré, professeur à l'École de service social de I'Université Laval, a procédé à la révision finale du texte. 
interne et diffuse dans la profession de redéfinir sa propre identité théorique et pratique, ces changements semblent aujourd'hui pouvoir mener à un savoir plus solide et original et à des fonctions sociales plus reconnues.

Cette constatation oriente le genre d'approche et le choix des contenus qui seront abordés dans cet article, pour essayer de donner une image suffisamment claire, bien qu'elle ne soit pas exhaustive, de la réflexion du service social sur sa propre réalité.

Naturellement, ce choix et les arguments que nous utiliserons se ressentent du point d'observation où s'est élaborée notre expérience pendant plusieurs années et du genre d'activité professionnelle que nous exerçons actuellement ${ }^{1}$.

Nous concentrerons donc nos réflexions sur quelques-uns des objectifs, des critères et des principes opérationnels d'un profil redéfini de la travailleuse ou du travailleur social, dans le contexte social institutionnel italien.

Nous chercherons plus précisément à retracer les nouveaux contours du " terrain d'action » du service social, d'abord comme résultante de l'évolution de la réflexion de la profession sur les changements historiques et institutionnels qui la touchent, pour en souligner ensuite l'importance et les contenus, à la lumière de récentes contributions théoriques.

Dans une deuxième partie, nous approfondirons deux aspects particulièrement liés à la spécificité pratique et théorique du service social italien:

- le genre de relation qui lie la travailleuse ou le travailleur social à l'organisation institutionnelle dont elle ou il dépend et les influences qu'un tel lien exerce sur la redéfinition de certains objectifs et principes opérationnels professionnels;

- le problème de la compatibilité ou de l'incompatibilité entre le but $d^{\prime}$ " aide» et l'exercice d'un " contrôle social » par le service social.

II s'agit de thèmes très débattus et particulièrement cruciaux dans la pratique quotidienne des intervenantes et intervenants, et qui constituent des domaines ouverts de recherche et d'expérimentation.

\section{ÉVOLUTION HISTORIQUE DES CONTEXTES DE PRATIQUE DU SERVICE SOCIAL EN ITALIE}

Pour rendre compréhensibles les orientations actuelles de la théorie et de la pratique du service social italien, il est nécessaire de reparcourir, d'une façon très sommaire, l'histoire de certaines transformations institutionnelles qui se réalisèrent à partir des années 70 .

À cette époque, on a assisté à une accélération considérable de la réflexion et de la théorisation du service social, aussi bien en ce qui con- 
cerne le refus du rôle culturel subalterne qui avait caractérisé la profession depuis ses origines que dans la recherche d'un «statut» théorique et pratique autonome.

À partir des années 70, en effet, il y a eu - enfin - la première réalisation de quelques-unes des réformes annoncées dans la Constitution de 1948, concernant, d'un côté, les problématiques liées à l'organisation de l'État et, de l'autre, le dépassement radical du système de réponses centré sur les organismes d'assistance.

Au cours des années 70 , on a institué les régions vers lesquelles furent décentralisées des tâches de programmation et de coordination générale, et, par la suite, de délégation aux communes pour la gestion directe d'une série de services au niveau local.

Quant au secteur institutionnel d'assistance, avant les années 70 il était composé de 40000 organismes répartis sur l'ensemble du territoire national. Ils constituaient un système compact, aussi bien public que privé, agissant en parallèle à l'État et aux organismes de gouvernement local. Ce système était centralisé et autoritaire, bureaucratisé et imperméable aux innovations techniques et scientifiques. II fournissait des prestations à des catégories "juridiques" de nécessiteux, suivant des critères moralistes et discrétionnaires.

Le réel dépassement d'une telle situation advint par la mise en marge des organismes d'assistance, en 1975, et par l'identification de nouveaux sujets institutionnels, habilités à programmer et à gérer des réponses adéquates aux multiples besoins des populations. On rattacha ces nouveaux sujets au système des autonomies locales, en particulier à la commune, à laquelle est dévolu le gouvernement d'une zone territoriale délimitée. La commune constitua la structure unifiante de toutes les réponses aux exigences de la vie civile locale des citoyens, par la gestion de services sociaux. Il s'agit toutefois de services se rapportant non seulement aux besoins d'assistance, mais aussi à ceux qui sont liés à d'autres domaines du social (santé, école, loisirs, habitation) et qui trouvent sur le territoire leur élément unifiant. Les « clients » de l'assistance sont devenus des " usagers » des services ${ }^{2}$.

Ainsi une grande partie des travailleuses sociales et travailleurs sociaux travaillant dans les vieux organismes d'assistance furent déplacés vers les organismes locaux.

Au début des années 80 , le réseau des nouveaux services s'étend (les centres de consultation pour les familles, les services territoriaux « de base ", etc., se répandent), et beaucoup d'autres intervenantes et intervenants sont intégrés dans les services (psychologues, éducateurs, auxiliaires familiales, etc.)

Le nouveau contexte constitue, pour le service social, un excellent cadre de développement de l'intervention sociale et communautaire, 
même si le chemin des réformes était et est difficile, rempli de problèmes, d'obstacles et de contradictions.

La confrontation du service social avec d'autres professions, la variété des options et des parcours politiques liés aux contextes locaux ainsi que le rôle plus dynamique des lieux de formation donnent de plus en plus de consistance au processus de redéfinition d'un profil professionnel pour la travailleuse ou le travailleur social. À ces facteurs s'ajoute l'instauration entre le travail pratique et la réflexion théorique de constantes modalités de comparaison ${ }^{3}$.

Ce qui paraît nouveau pour le contexte italien, c'est cette autonomie de la profession, et en même temps son interdépendance avec les contextes, les problématiques sociales et les autres professions, en conjonction avec la recherche continue d'une élaboration théorique qui s'inspire de cette interdépendance.

En outre, la production théorique du service social trouve sans doute une stimulation et un soutien plus importants dans le cadre des récentes modifications structurales du système de formation, modifications liées à la reconnaissance formelle, en 1987, du titre professionnel par l'État. Maintenant, la formation des travailleuses sociales et des travailleurs sociaux est exclusivement prise en charge par les universités, dans le cadre $d^{\prime}$ " écoles orientées vers des buts particuliers pour travailleuses sociales et travailleurs sociaux».

\section{LES COORDONNÉES DU NOUVEAU CHAMP D'ACTION}

Le dépassement de l'optique d'assistance, dans le cadre des réformes, en mettant fin à la séparation et au caractère marginal du système institutionnel de réponse aux besoins sociaux des citoyens, a permis de mieux voir les liens réels existant entre la naissance-transformation des besoins et les conditions sociales ambiantes qui contribuent à les créer. Le champ d'action traditionnel du service social - la relation entre les personnes et le milieu - assume ainsi des connotations plus précises et aussi plus complexes. Le «milieu» devient alors aussi bien l'ensemble des conditions sociales générales, le lieu des choix politico-économiques et institutionnels du pays que la micro-réalité de la communauté territoriale où la traduction de tels choix en infrastructure de services va produire des interférences avec les processus sociaux réels, les styles de vie, les problèmes, la potentialité, les intentionnalités de la population locale.

Il est extrêmement utile, pour le service social, d'appeler un tel milieu un " éco-système ${ }^{4}$ ", dont l'équilibre est la résultante d'un entrelacement de facteurs personnels et environnementaux, parce que cette désignation met en évidence que : 
- les interruptions, le mauvais fonctionnement, les déviations dans le rapport personne-milieu peuvent être gérés et ne produisent pas de "pollution » dans la mesure où l'éco-système exprime et développe des ressources adéquates aussi bien personnelles et individuelles que sociales;

- la plus grande visibilité de la continuité entre la dimension individuelle et la dimension sociale, aussi bien des problèmes que des ressources, permet d'agir dans l'intérêt de la collectivité, sans nier les différences individuelles, sociales, territoriales; mais au contraire, en les utilisant comme potentialité $d^{\prime}$ 'innovation et de transformation, au lieu de détachement et d'exclusion, c'est-à-dire en promouvant de nouvelles autonomies comme moteur, en même temps de transformation personnelle et sociale;

- pour produire un changement en matière d'émancipation individuelle et sociale, il faut recomposer les rôles et les responsabilités $d^{\prime}$ une multiplicité d'acteurs (y compris les destinataires des interventions), parmi lesquels «la présence technique assume une fonction de facilitation du processus plutôt que d'organisation des offres sociales " (Ferrario et Gottardi, 1987: 34). En outre, tous les « sujets » qui composent le tissu communautaire: groupes, associations, représentants sociaux et politiques, forces syndicales, associations volontaires, etc., acquièrent de l'importance et un rôle de protagonistes;

- pour compléter ce qui a été dit jusqu'ici, les actions de transformation réalisées par les institutions et par les intervenantes et intervenants ne peuvent pas toucher seulement la personne ou seulement l'environnement, parce que cela produirait fatalement une séparation entre la personne et son milieu et la rendrait dépendante de ce milieu.

En ce qui concerne la spécificité de l'intervention du service social, on en déduit l'adoption d'une approche unitaire aussi bien sur le plan de la lecture des problèmes dont il s'occupe (ils sont presque toujours soit de la personne, soit du milieu) que sur celui de la focalisation de l'intervention (qui prévoit des actions directes, avec cependant des instruments différents, aussi bien avec la personne qu'avec le milieu).

Avec la réalisation des réformes, toutefois, un troisième sujet vient faire partie, à plein titre, de la redéfinition concrète du champ d'action du service social. C'est l'organisme local - la commune - qui gère les services auxquels sont rattachés la plupart des travailleuses sociales et des travailleurs sociaux. La commune est la représentante directe de la communauté territoriale locale (qui élit les conseillers communaux et qui a le droit et le devoir d'en contrôler le travail). Elle constitue un sous-système agissant aussi bien avec les citoyens eux-mêmes qu'avec la complexe réalité territoriale. Les services qu'elle gère deviennent une ressource 
appartenant à la communauté locale (même s'ils sont certainement plus artificiels et par conséquent plus «polluants » que les activités spontanées de la communauté elle-même).

Ainsi, le caractère unitaire des interventions du service social prend la forme de processus de connexion entre la personne, le territoire et I'organisation de services.

$C^{\prime}$ est justement le fait de «maintenir unis» ces trois éléments qui permet à l'intervenant ou à l'intervenante de poursuivre efficacement son but de promotion de l'autonomie. L'augmentation de ressources sociales, et de prestations de services en particulier, ne répond pas en soi aux situations de besoin; au contraire, elle crée facilement une dépendance et donc engendre de nouveaux besoins. Ce n'est que si les sujets développent une capacité de "gérer » de telles ressources (et non pas simplement $d^{\prime}$ en " jouir ", de les consommer) que celles-ci deviennent une occasion et un instrument de croissance personnelle et sociale.

Naturellement, la condition nécessaire est la reconnaissance de la subjectivité (soit individuelle, soit collective) qui fait des personnes en état de besoin non pas de simples destinataires de prestations, mais des interlocuteurs en puissance, en mesure de modifier les rapports existants et d'acquérir de l'autonomie.

En ce sens, par exemple, la reconnaissance de la communauté locale, comme sujet, affirmée par le gouvernement local de la commune dans la production de services, comporte aussi le droit et la responsabilité de tous les sujets de la communauté elle-même de contribuer à la réelle prise en charge de leurs propres problèmes et au développement de ressources pour leur faire face, y compris de prendre part avec l'institution à l'élaboration des processus de conjugaison des responsabilités publiques avec les droits des citoyens.

En résumé, le fait de «maintenir unis » les trois sous-systèmes signifie pour le service social:

- se placer au croisement des besoins et des ressources aussi bien individuels que sociaux:

- mettre en évidence les potentialités de transformation (aussi bien dans les besoins que dans les ressources), en réduisant la dépendance surtout institutionnelle et en promouvant la reconnaissance comme sujets des personnes et des institutions, aussi bien que leur responsabilité;

- promouvoir une «culture des ressources» (institutionnelles ou non, personnelles et sociales) qui prévoit et développe une capacité 
$d^{\prime}$ autonomie, qui à son tour libère des ressources nouvelles et plus authentiques.

L'intervention du service social aura donc une orientation promotionnelle et éducative plutôt que thérapeutique et curative, c'est-à-dire capable d'amorcer des processus d'apprentissage social pour et par I'usage, la promotion et la transformation de ressources personnelles et sociales $^{5}$.

On pourrait dire, en effet, que le travail de l'intervenante et de l'intervenant est beaucoup plus grevé par un manque excessif de ressources que par l'excessive gravité des problèmes.

La fonction promotionnelle et éducative qualifie constamment l'intervention de la travailleuse sociale et du travailleur social, aussi bien quand elle ou il s'occupe directement de la demande individuelle d'aide que lorsqu'elle ou il travaille plus directement avec le territoire ou avec sa propre organisation institutionnelle. Cette multidimensionnalité caractérise la profession, quel que soit le service dans lequel elle est exercée. Elle devient pratiquement réalisable à partir du moment où on adopte une logique de projet global qui donne un sens et intègre les diverses actions et relations, et qui est capable de stimuler une pensée stratégique, transformatrice, dans tous les sujets en cause, en se référant à des objectifs concrets communs.

Il serait maintenant nécessaire de réfléchir plus avant sur l'articulation détaillée du rôle des sous-objectifs, des modalités d'intervention professionnelle dans un domaine si vaste et complexe dont nous avons tout juste esquissé les contours.

Nous pourrons nous arrêter un instant seulement pour déterminer les caractéristiques d'un des sous-systèmes de relations que l'intervenante ou I'intervenant instaure dans son action professionnelle, celui qui concerne son lien avec l'organisation institutionnelle de laquelle elle ou il dépend.

Les relations avec les personnes usagères et avec le contexte communautaire ont été souvent étudiées et elles ont donné lieu à de nombreuses publications, aussi bien en Italie qu'à l'extérieur de l'Italie.

Le rapport avec l'organisation est un domaine de recherche nouveau en service social. C'est pourtant la dimension avec laquelle la profession se mesure quotidiennement, y compris à travers des expériences parfois pleines de fortes contradictions. Sa complexité est liée au fait que l'organisation elle-même devient toujours plus complexe et que l'institution est en même temps lieu et sujet d'un mandat concret pour la travailleuse sociale et le travailleur social, ressources et obstacles pour son travail professionnel. 


\section{LE TRAVAIL DE LA TRAVAILLEUSE SOCIALE ET DU TRAVAILLEUR SOCIAL AU SEIN DE L'ORGANISATION ET POUR L'ORGANISATION INSTITUTIONNELLE}

Dans la partie historique, nous avons déjà fait allusion au processus, encore en cours et plein de contradictions, de territorialisation, d'ouverture, de démocratisation des organisations publiques; ce processus a comporté un accroissement du nombre de sujets interlocuteurs, aussi bien internes (par exemple, la multiplication et la diversification des professionnelles et professionnels) qu'externes (les citoyennes et citoyens usagers et l'ensemble de la communauté territoriale) aux structures de service.

Le lieu institutionnel où la travailleuse sociale et le travailleur social exercent leurs fonctions s'en ressent évidemment. Les qualités professionnelles et l'intervention d'aide aux usagers elle-même prennent des connotations différentes.

$\mathrm{Si}$, d'un côté, la travailleuse sociale et le travailleur social partagent avec d'autres professionnels des services une série de fonctions portant sur l'élaboration de plans, le contrôle, la gestion d'interventions sur le plan de l'organisation, de l'inter-organisation et du territoire, de l'autre ils se différencient aussi des autres intervenantes et intervenants par la position qu'ils occupent en général au sein des niveaux d'organisation et par le genre de rapport qui les lie à l'organisation.

Les recherches de ces dernières années ${ }^{6}$ confirment l'existence d'un lien particulièrement intense entre le service social, l'organisation de service et les politiques sociales. Le service social s'avère être, parmi les différentes professions en cause, celle qui sans aucun doute a le plus de " capacité $d^{\prime}$ interprétation des processus institutionnels, [...] de dialogue avec le personnel politique et surtout [...] d'esprit d'adaptation à des situations peu structurées et "devant être construites» (Neve et Niero, 1990: 31).

Réfléchissons un moment sur les influences réciproques entre ce lien complexe avec l'organisation institutionnelle et la nature, les buts, les modalités d'action du service social.

\section{LES FONCTIONS DE «LIAISON» ENTRE SERVICES ET COMMUNAUTÉ LOCALE}

En relation avec ce qui a été dit jusqu'à présent, on peut définir la travailleuse sociale ou le travailleur social comme la personne qui "agit en même temps dans une dimension personnelle et dans une dimension institutionnelle, non seulement dans le but d'une meilleure correspon- 
dance de la réponse à la demande (une réponse plus personnelle), mais aussi pour une transformation de la demande en ressource potentielle (une demande plus sociale) » (Ciolfi et Milana, 1983: 56). La définition insiste sur le fait que, en ce qui concerne les services, ils doivent être (ou devenir) quelque chose qui est "au service de... », c'est-à-dire dont le but est de faire progresser la communauté des citoyens, non pas de distribuer des réponses. En d'autres mots, les organisations de service devraient et peuvent assumer une fonction fondamentale de liaison avec la communauté locale, vu qu'elles représentent l'espace structurel institutionnel d'échange avec les individus du territoire pour la transformation aussi bien des problèmes que des réponses.

Nous pensons que la travailleuse sociale et le travailleur social occupent cet espace, ou plutôt qu'ils sont les techniciens de ce genre de rapport (donc non pas de $n^{\prime}$ importe quel rapport individu-structure) (Ciolfi et Milana, $1983: 58$ ).

En effet, si le but professionnel de l'intervenante ou de l'intervenant consiste à faire obstacle à la dépendance et à la marginalisation provoquées par l'état de besoin, en mettant en valeur et en activant les ressources, même restantes, d'autonomie des personnes, elle ou il doit agir de telle manière que, par exemple, I'accès lui-même de l'usager au service (qu'il soit spontané ou imposé de quelque façon) se transforme en une expérience $d^{\prime}$ autonomie, qui constitue un espace où déjà la définition elle-même du problème soit faite " avec » la personne et puisse être transformée en un processus d'apprentissage social.

À l'inverse, la simple fourniture de prestations (dans une logique de " demande-réponse ») tend en soi à créer une dépendance, qui est en plus institutionnelle, et "il est facile que la dépendance institutionnelle devienne chronique» (De Leonardis, 1990: 53).

En d'autres mots, I'habileté de la travailleuse sociale ou du travailleur social à instaurer un bon rapport avec les personnes, bien qu'elle soit nécessaire, n'est pas suffisante pour que celles-ci sachent "gérer » les prestations et non pas simplement en «jouir». II faut que cela advienne dans un contexte de réelle liaison où l'organisation, elle aussi, soit disposée à subir des contrôles et à confronter des objectifs et des modalités de réponses avec les individus de la communauté. II doit donc s'agir d'une organisation capable de fournir des services et des prestations suffisamment élastiques, ouverts, intégrés, de telle sorte qu'ils deviennent de plus en plus des instruments de changement et d'avancement social et culturel.

Ainsi, "personnaliser » les réponses signifie aussi pour la travailleuse sociale ou le travailleur social agir directement sur l'organisation, par exemple pour «inventer» ou rendre plus flexibles les prestations (telles 
que I'apport de contributions économiques, de services d'assistance domiciliaire, etc.) ou pour amener des choix d'intervention territoriale ouverte plutôt que marginalisatrice (comme par exemple chercher des familles auxquelles confier les enfants plutôt que de les placer dans une institution), pour promouvoir la condition de sujet et la capacité de réflexion de la même organisation ${ }^{7}$, etc.

\section{LE PRINCIPE DE LA GLOBALITÉ}

Ce besoin de flexibilité, de changement au sein des organisations qu'éprouve la travailleuse sociale ou le travailleur social est par ailleurs lié aussi à la nature des problèmes dont elle ou il s'occupe le plus souvent, et en détail. Ceux-ci se présentent comme « un fait de la vie: un enfant qui se drogue, une personne âgée qui ne peut plus vivre seule, etc. De cette façon, le problème est encore intact dans sa globalité, il n'a pas été décomposé suivant les diverses spécialisations » (Ciolfi et Milana, 1983: 57). Cela signifie que bien souvent ces problèmes ne sont pas encore ou ne sont pas complètement codifiés par le système institutionnel, soit à cause de la grande diversité dans laquelle on les vit et avec laquelle ils se manifestent, soit à cause de la tendance du système à les classifier comme sanitaires, psychiatriques ou psychologiques, etc., au lieu de les considérer dans leurs interrelations personnelles et environnementales.

C'est pourquoi souvent les réponses ne sont pas prêtes; il faut les former ou les inventer. Cela comporte pour la travailleuse sociale ou le travailleur social un travail constant de mise en œuvre ou de modification de procédures, de programmes, de ressources, aussi bien à l'intérieur qu'à l'extérieur de l'organisation. Pour que les réponses puissent vraiment promouvoir l'autonomie des personnes, afin de libérer de nouvelles ressources, il est nécessaire que de telles réponses soient globales, c'est-à-dire qu'elles ne décomposent pas artificiellement les problèmes des personnes jusqu'à les rendre méconnaissables à elles-mêmes.

$C^{\prime}$ est ici que l'action de l'intervenante ou de l'intervenant envers I'organisation devient une tâche fondamentale de connexion, de coordination, d'intégration de ressources, d'instruments et d'informations aussi bien à l'intérieur qu'à l'extérieur du système d'organisation institutionnel.

La transparence, la fluidité des rapports entre les services et leur réelle complémentarité représentent la qualité structurale requise pour I'efficacité des interventions et en même temps un des objectifs (pour la travailleuse sociale ou le travailleur social) de "promotion » de cette ressource communautaire qu'est l'organisation de services. 


\section{LE TRAVAIL DE TRANSFORMATION DE LA «LIMITE » INSTITUTIONNELLE}

Pour compléter la vue d'ensemble du travail de l'intervenante ou de l'intervenant avec et pour l'organisation institutionnelle, nous ne pouvons mettre de côté le fait que la travailleuse sociale ou le travailleur social, plus que d'autres professionnels, est, en quelque sorte, une intervenante ou un intervenant "de frontière", car elle ou il doit tenir compte d'une série de frontières et de limites surtout institutionnelles.

Pour la travailleuse sociale ou le travailleur social, le fait de se trouver sur le point de jonction entre le service et la communauté territoriale veut dire vivre tout le caractère tragique, mais aussi la richesse du rapport entre citoyen et institution.

Il s'agit là d'un rapport de limite réciproque: I'institution marque concrètement une limite pour l'usager (elle impose des règles, exige certains comportements, elle ne peut pas donner une réponse à tout et à tous, elle tend à exclure les individus qui ne peuvent pas être traités...), de même que l'institution est limitée par la condition de sujet ainsi que par l'intentionnalité de l'usager et de la collectivité.

$\mathrm{C}^{\prime}$ est justement sur cette limite que se joue, matériellement, jour après jour, le défi de la compatibilité entre les critères universalistes et la sauvegarde des différences et des particularités individuelles; entre le caractère abstrait des normes, le caractère obligatoire du droit et la condition de sujet ainsi que la responsabilité des différents interlocu-teurs, qu'ils soient institutionnels ou non; entre ce qui est désirable (et juste) pour l'intervenant ou l'intervenante et ce qui est matériellement faisable.

Ici, certaines contradictions sociales, ou encore les ambivalences des institutions elles-mêmes, tendent à se traduire en demandes pressantes à la travailleuse sociale ou au travailleur social d'assumer les tâches les plus disparates, de s'occuper des problèmes les plus dramatiques et les moins solubles et d'être d'une disponibilité sans limites.

Pourtant, c'est justement la matérialité de l'institution - son caractère concret - qui permet de travailler avec les moyens qu'on a et non avec les désirs qu'on aurait. Elle y oblige même. Sur ce plan, I'intervenante ou l'intervenant peut jouir d'une certaine autonomie: ce n'est qu'en connaissant et en employant (et en apprenant à employer) les conditions subjectives et structurales réelles, les ressources humaines, culturelles, matérielles, économiques existantes qu'elle ou il peut en expérimenter un usage différent, combiné à un recours plus efficace à des ressources extérieures (par exemple, les ressources communautaires), tout en agissant pour un changement des règles du jeu internes à l'organisation de services, une définition différente des objectifs, etc. ${ }^{8}$. 
En somme, la limite concrète de l'institution, comme beaucoup $d^{\prime}$ autres limites, peut devenir source d'énergie absorbant et alimentant une " culture des ressources », parce qu'elle-même engendre des possibilités $d^{\prime}$ expérimentation, $d^{\prime}$ « apprentissage social», aussi bien pour l'intervenante ou l'intervenant que pour tous les sujets concernés.

\section{AIDER ET CONTRÔLER: UNE CONTRADICTION?}

Un des aspects peut-être les plus discutés de la profession par rapport au contexte opérationnel actuel concerne le rôle $d^{\prime}$ " aide » de la travailleuse sociale ou du travailleur social en relation avec sa compatibilité avec les frontières du contrôle social.

Ce qui pousse à cette remise en discussion, c'est l'augmentation progressive des demandes adressées aux travailleuses sociales et aux travailleurs sociaux pour qu'ils exercent des fonctions de contrôle social (par exemple, à l'égard du comportement déviant de mineurs et d'adultes, de situations familiales fortement conflictuelles, de la toxicomanie, de I'immigration, etc.) en plus du fait d'être eux-mêmes « objets » de contrôle (par exemple, de la part de l'autorité judiciaire, des services eux-mêmes, de I'opinion publique, etc.).

La profession reflète souvent une image de disponibilité inconditionnée (presque comme ingrédient nécessaire pour pouvoir vraiment aider), avec les déceptions et les frustrations d'une aide irréalisable, avec le dilemme d'accepter ou de refuser des mandats de contrôle même "forts " sur les personnes. Souvent, l'aide est considérée comme étant le bon côté de l'intervention et le contrôle comme le mauvais côté.

Convaincue de la nécessité de soustraire ces concepts à toute interprétation idéologique, et cela autant que possible, nous voudrions parler brièvement de quelques réflexions sur cette question.

Il est incontestable que $n^{\prime}$ importe quelle société organisée a besoin de produire des mécanismes de contrôle, quoique dans des formes et avec des significations différentes selon le genre de société et de modèle idéologique et culturel sur lequel elle se base. En effet, il y a toujours besoin de contrôler, c'est-à-dire de régler, de maintenir unie la société pour en éviter la désagrégation. Et cela advient, par exemple, grâce aux processus de socialisation qui mènent à des comportements respectueux des normes prévalentes, mais aussi grâce à des mécanismes de représsion pour défendre la collectivité contre des individus ayant un comportement déviant; ou encore par des procédés de régulation, de sélection, de limitation, dans le but difficile à poursuivre d'une sauvegarde aussi bien de l'identité individuelle que de l'ordre, de l'intégration sociale, etc. De très nombreuses études, surtout sociologiques, ont été publiées en ce sens. 
Il est aussi évident que dans des sociétés aussi complexes que les nôtres, les personnes déléguées à l'exercice de ces contrôles ne peuvent plus être assimilées au stéréotype de l'unique pouvoir souverain qui établit les normes et exige obéissance et fidélité des sujets. C'est comme si aujourd'hui ce pouvoir s'était brisé et avait été partagé entre une multiplicité d'organismes et d'individus jusqu'à s'insinuer dans les microcontextes et dans les relations sociales, pour enfin devenir « forme de la communication " (Pitch, 1987). Il existe une pluralité de personnes - et de pouvoirs - de contrôle et il y a aussi différents genres et usages du contrôle social, fortement entrelacés dans la vie quotidienne des individus, avec des interactions qui lient les citoyens entre eux et aux institutions.

On pourrait dire plutôt que plus on enlève aux personnes et aux contextes de vie le pouvoir d'autocontrôler leur propre existence et leur vie en commun, plus ils doivent subir des contrôles arbitraires qu'euxmêmes n'ont pas contribué à déterminer: ils ont "perdu le contrôle".

En ce sens, le système institutionnel $d^{\prime}$ 'assistance en place avant les réformes des années 70 est un exemple probant. Il constituait, en effet, un monde séparé, par sa structure, aussi bien de la vie réelle des personnes (il basait les interventions sur l'appartenance des personnes à des catégories juridiques abstraites) que de l'ensemble des politiques sociales (amenant ainsi le stéréotype que les causes des problèmes sociaux dépendent de chaque personne).

En outre, à l'intérieur même d'un tel système s'effectuait une sorte de séparation entre les interventions d'aide - gérées par les organismes de véritable assistance aux nécessiteux - et les interventions de contrôle, gérées par les « institutions totales », c'est-à-dire l'asile d'aliénés, les institutions fermées ou "spéciales", etc.

Ces dernières avaient, en effet, la tâche de contenir, en les réprimant, les comportements déviants ou irrécupérables. Cependant, en réalité, cette séparation entre celui qui avait la tâche d'aider (c'est-à-dire les organismes d'assistance) et celui qui avait la tâche de contrôler était seulement fictive et soutenue par une certaine idéologie de l'État social. En effet, les organismes d'assistance exerçaient, eux aussi, un contrôle beaucoup plus sournois et implicite, mais non moins fort, sur les personnes assistées, soit parce que l'accès à ces organisme "spéciaux » marquait les personnes comme des individus "différents ", de séries B, soit parce qu'on leur demandait essentiellement et seulement $d^{\prime}$ adhérer à des normes morales et juridiques (qu'elles-mêmes n'avaient pas contribué à créer!), sous peine de se voir refuser toute aide et de se faire envoyer dans des institutions répressives. À l'inverse, nous avons, par exemple, l'expérience du démantèlement des asiles psychiatriques qui a mené à la loi 180/78 où l'institution elle-même, qui contrôlait si fortement les patients, $\mathrm{n}^{\prime}$ a pas nié la possibilité de sa transformation en processus de libération et d'aide 
(aidée en cela par les patients eux-mêmes qui, appuyés par les intervenantes et intervenants, sont devenus "impatients ${ }^{10}$ ).

Cette référence socio-institutionnelle nous fait réfléchir, $d^{\prime}$ un côté, sur le fait que:

- il est bien différent - surtout en ce qui concerne les effets sur les destinataires - d'exercer des contrôles explicites et visibles ou, au contraire, implicites, dissimulés, en les faisant passer pour une " aide";

- une aide masquée par un paternalisme et une fausse neutralité (comme celle exercée de fait par les organismes d'assistance) cache des mécanismes de contrôle destinés à empêcher la potentialité des personnes, par conséquent à créer une dépendance, parce qu'ellesmêmes ne sont plus en mesure de les reconnaître; dans un certain sens, on leur enlève la capacité de penser.

D'un autre côté, il s'agit d'un exemple probant du processus par lequel une institution - $n^{\prime}$ importe quelle institution - dans la mesure où elle est séparée du contexte civil, humain et culturel auquel elle appartient (parce que «fermée », imperméable aux contrôles externes, soutenue par des logiques de prédéfinition rigide de besoins et de réponses) produit des rapports tendant davantage à la renforcer comme institution qu'à " céder » le pouvoir aux citoyens.

De nos jours, une grande partie de ces pouvoirs (d'aide et de contrôle des anciennes institutions) font de nouveau partie des compétences de l'organisme local et sont de nouveau répartis entre les institutions (plus ouvertes, plus perméables, etc.) et les sujets collectifs et individuels de la communauté territoriale, qui acquiert ainsi des droits et une responsabilité, du moins présumés, d'autogestion (et donc d'autocontrôle).

Le fait que les mêmes services de l'organisme territorial doivent s'occuper aussi de ces situations considérées autrefois comme "spéciales" (maladie mentale, comportement déviant, etc.) sans pouvoir recourir aux institutions totales (parfois même pas à la prison ${ }^{11}$ ) signifie:

- un déplacement des systèmes de contrôle social $d^{\prime}$ une logique de contrainte, d'imposition sur les personnes vers une logique de prédisposition, de construction d'instruments et processus sociauxinstitutionnels visant à intégrer les personnes dans leur propre milieu de vie;

- reporter, donc, dans les rapports sociaux et interpersonnels le caractère tragique, les conflits d'une vie avec le comportement déviant et la diversité; ce qui, notamment, augmente la nécessité de dispositifs de tutelle et de soutien pour les sujets les plus exposés (y compris les intervenantes et intervenants); 
- mettre donc en discussion nos représentations mentales (c'est-à-dire celles de tous et toutes), nos modèles d'acceptation/non-acceptation du "différent», en nous posant le problème d'activer des transformations, aussi bien environnementales que culturelles (des institutions, de la communauté, des autres personnes...) dans une logique de réciprocité avec les changements que nous voulons obtenir des personnes ayant un comportement déviant.

En outre, la territorialisation et la ramification des services et des interventions, qui tendent ainsi à pénétrer jusque dans les plaies les plus intimes de la vie personnelle et familiale, peuvent même devenir envahissantes, polluantes, justement dans le sens qu'elles contrôlent, en les manipulant, les personnes les plus faibles (par exemple, dans le domaine des rapports parents-enfants, du contrôle de la sexualité, de la procréation responsable, etc.); pourtant, il s'agit en même temps d'une garantie de disponibilité et de réponse aux exigences effectives des citoyens.

En somme, dans la pratique quotidienne des services et des interventions, il est très difficile de distinguer où l'aide finit et où le contrôle commence (avec toutes ses différentes significations), aussi parce que, de toute façon, beaucoup d'usagers ont besoin d' « apprendre " à se contrôler et à s'administrer eux-mêmes.

Le problème prend alors une nouvelle orientation et consiste à savoir quel genre de contrôle nous acceptons d'exercer (et quel genre de contrôle nous refusons) et dans quelle mesure nous en sommes conscients.

$S^{\prime}$ il est vrai qu'il existe des contrôles plus clairs et explicites et d'autres moins visibles et plus sournois, il existe aussi des contrôles qui sont plus acceptés par les usagers eux-mêmes et par l'opinion publique (plus fréquents dans le domaine de la santé: par exemple, le patient qui subit une intervention chirurgicale est entièrement entre les mains du médecin) et d'autres qui sont moins acceptés (par exemple, le fait d'éloigner les enfants des familles). II y a des contrôles qui empêchent et nient grandement la liberté des personnes; il y en a d'autres qui limitent la liberté individuelle, mais dans le but $d^{\prime}$ 'éduquer les personnes en matière $d^{\prime}$ autonomie et de respect des libertés d'autrui (un contrôle typique est celui des parents qui contrôlent leur enfant pour qu'il grandisse et apprenne l'autocontrôle).

Nous ne sommes certainement pas en train de dire qu'un contrôle rigoureux, même répressif sur les personnes (droit dont est titulaire l'autorité judiciaire) doit être éliminé: ce serait une pure utopie. Nous voulons dire qu'il doit être le plus possible remplacé ou au moins accompagné $d^{\prime}$ un autre genre de contrôle que nous appelons sur les processus. Ces processus individuels, sociaux, institutionnels concourent à déterminer, et peuvent donc aussi prévenir et réabsorber, en le réglant, le comporte- 
ment déviant. Cela ne signifie rien d'autre qu'amorcer des processus $\mathrm{d}^{\prime}$ aide-contrôle dans le sens de promotion de conscience, de responsabilité personnelle, d'intégration entre différents sujets, de renforcement des réseaux spontanés d'aide, de libération des ressources, etc. En d'autres termes, plus les interventions s'orientent vers la prévention et la promotion, plus un contrôle sur les processus est réalisable; plus on insiste sur les soins et la répression et plus on effectue un contrôle sur les personnes. Le dilemme de l'apparente dichotomie aide/contrôle réside plutôt dans la difficulté et dans les nombreuses contradictions que comporte souvent le fait de les gérer correctement et consciemment (c'est-à-dire sans glisser dans des contrôle arbitraires sur les personnes).

Si on l'analyse à fond, on peut dire que la tâche institutionnelle de la travailleuse sociale ou du travailleur social est toujours aussi bien d'aide que de contrôle, même si elle n'est pas explicite.

Il est vrai toutefois que dans le concept de contrôle, ou mieux dans la relation inséparable aide/contrôle, il y a une grande ambiguité, attribuable aux forts contenus émotifs suscités par de tels concepts (par exemple, nos expériences en ce qui concerne l'autorité, le pouvoir, la liberté, etc.) et aux stéréotypes culturels qui tendent à scinder, par exemple, les rôles: féminin (aide maternelle) et masculin (porteur d'autorité et de contrôle ${ }^{12}$ ).

Et il est très facile que l'aide se traduise en imposition ou prescription, que l'autorité professionnelle se transforme en autoritarisme, que le pouvoir «de..." devienne pouvoir «sur...»; en somme, que le contrôle sur les processus, du fait qu'il requiert une grande capacité d'autocontrôle, dégénère en contrôle sur les personnes.

\section{CONCLUSION}

Cette vision, à peine abordée, des concepts de contrôle et d'aide, de la nécessité-possibilité de leur conjugaison à l'intérieur de l'intervention professionnelle se situe sur une ligne de continuité avec les principes et les objectifs généraux du service social dont nous parlions au début. Elle en souligne la complexité et met en évidence la nature problématique de sa réalisation et la nécessité $d^{\prime}$ adopter des stratégies efficaces qui permettent de concilier ce qui est désirable avec ce qui est faisable. Ainsi, des interventions d'aide, même connotées de fortes valeurs de régulation et de contrôle, deviennent indispensables pour pouvoir "maintenir unies", dans un processus unitaire à plusieurs dimensions, les personnes - avec les sujets du territoire - avec les organisations de services. Cela exige des opérations de continuelle négociation, une capacité de coordination et d'intégration d'interventions, destinées à promouvoir des ressources et à 
rendre responsables des sujets de la communauté, à développer et à renforcer le «tissu» social.

Donc, le travail social ne peut pas être un travail solitaire et encore moins improvisé: le partage nécessaire avec les autres sujets, institutionnels ou pas, est réalisable dans une logique de projet global qui garantit aux interventions cohérence et clarté, suivant des buts chaque fois confrontés et vérifiés.

$C^{\prime}$ est ainsi qu'une profession éminemment pratique et orientée vers I'action concrète ne refuse pas une réflexion "forte " qui oriente et donne un sens à l'action. Au contraire, elle s'en nourrit et en tire un enseignement fondamental pour améliorer la compréhension et la gestion des problèmes sociaux.

\section{Notes}

1. L'auteure fait ici référence à ses vingt ans d'activité dans le domaine de l'enseignement du service social, de la formation pratique des étudiantes et étudiants, de la participation à des recherches, et à ses fréquents rapports avec les intervenantes et intervenants dans le cadre de consultations et de stages de perfectionnement et de formation continue.

2. En Italie, avec les réformes des années 70 justement, on passe du terme « client » à celui $d^{\prime}$ " usager», pour souligner le fait qu'une personne est titulaire d'un droit à recevoir un service public en tant que citoyen. Le mot "client " met davantage en évidence l'aspect de paternalisme, de sujétion et de chronicité. Il est employé en politique avec un sens plutôt péjoratif: "Personne qui se met à disposition d'un personnage influent, surtout politique, pour obtenir en échange faveurs et protection " (Devoto-Oli, Vocabolario della lingua italiana, Le Monnier, Milan, 1979).

3. Quelques séminaires et congrès (Milan en 1984 et 1985, Vérone en 1985, Venise en 1987 et Trieste en 1990) représentent des étapes significatives de ce parcours de nouvelle élaboration et de renforcement théoriques et professionnels.

4. L'approche écologique et systémique appliquée au service social a fait sa première apparition en Italie au début des années 70 dans deux séminaires tenus par deux experts américains. Quelques auteurs italiens font maintenant référence à des ouvrages de C. Germain et de H. Goldstein (Dal Pra Ponticelli, 1987).

5. Référence explicite à la "théorie de l'apprentissage social " et aux nouvelles orientations cognitivistes. Sur ce point, voir Dal Pra Ponticelli, 1985, de même que Ferrario et Gottardi, 1985.

6. Citons parmi les plus significatives :

IRESS, Région Émilie-Romagne, 1980, Assistente sociale, quale futuro ? Patron, Bologne. Deux volumes.

RUGERI, F., 1982, Assistente sociale. Processi di cambiamento, politica dei servizi, Nuova Guaraldi, Florence.

MINISTÈRE DE L'INTÉRIEUR, Direction générale des services civils, 1984, L'assistente sociale nel nuovo assetto dei servizi : problemi e prospettive, Rome. 
ÉCOLES DE SERVICE SOCIAL DE VENISE ET VÉRONE, 1990, Rapporto sulle professioni sociali 1990 , région Veneto. Cette dernière publication présente les résultats les plus récents d'une recherche longitudinale commandée en 1976 par la région Veneto et qui constitue une observation continue des politiques, des services et des intervenantes et intervenants du secteur. Des réflexions tirées de cette recherche ont été présentées dans deux autres publications antérieures:

BERNARDI, L., J. DE SANDRE et S. GIRALDO et al., 1980, Composizione e organizzazione del lavoro sociale, Écoles de service social de Venise et Vérone.

Bernardi, L., J. De SANDre et S. Giraldo et al., 1985, Professionalità sociali e innovazione, Cappelli, Bologne.

7. Il est typique des organisations bureaucratiques de demander de ne pas réfléchir: ce qui est plutôt exigé, $\mathrm{C}^{\prime}$ est le respect et l'accomplissement formel de la norme, qui tend ainsi à devenir une fin en soi.

8. Une intéressante synthèse de Valerio Ducci dans Coordinamento Nazionale Docenti di Servizio Sociale, I/ servizio sociale come processo di aiuto, F. Angeli, Milan, 1987, met en évidence, tout en citant Crozier et Friedberg, que I'organisation n'est pas un tout compact parfaitement rationnel et immuable; "les règles [...] peuvent être remises en discussion, négociées à nouveau », par conséquent chaque acteur de l'organisation a des possibilités (plus les valeurs et les objectifs poursuivis par l'organisation elle-même peuvent être partagés et plus grandes sont ces possibilités) d'employer ou de gagner des espaces de manœuvre pour modifier des normes, des procédures, etc., même dans des situations passablement contradictoires.

9. L'histoire du démantèlement des asiles psychiatriques est née dès le début des années 60, avec l'équipe psychiatrique de Franco Basaglia, d'opérations apparemment banales telles que : redonner aux patients leurs propres objets personnels, la possibilité de prendre la parole dans les assemblées de service, libérer les sentiments...; c'est-à-dire qu'on fait progressivement un usage différent de " [...] ce qu'il y a [...] mais l'ensemble perd fixité, se décompose et change [...]. Une porte faite pour être fermée est employée, mais activement employée, pour être ouverte " (De Leonardis, op. cit.).

10. Il s'agit d'une expression suggestive de O. De Leonardis, 1990:69.

11. Depuis 1975 , il y a eu une série de lois de réforme dans le domaine pénal (et civil) aussi bien pour les mineurs que pour les adultes. Ces lois prévoient, par exemple, la possibilité de peines autres que la prison, même par l'intermédiaire de la collaboration entre la magistrature et les services sociaux, dans une optique - que nous jugeons avancée d'accentuation du « traitement » par rapport à la " punition » et d'un plus grand partage de responsabilités entre juges et intervenantes ou intervenants.

12. En Italie, du moins, la très grande majorité des professions d'aide, et en particulier le service social, est formée de femmes. 


\section{Références bibliographiques}

BERNARDI, L., I. DE SANDRE et al. (1980). Composizione e organizzazione del lavoro sociale. Scuole di servizio sociale di Venezia e Verona.

BISLERI, C. et S. GIRALDO (1990). "Tirocino e professionalità nell'evoluzione del servizio sociale italiano ", E. NEVE et M. NIERO (dir.), II tirocinio. Modelli e strumenti dall'esperienza delle scuole di servizio sociale italiane. Milan: Franco Angeli.

Busnelli, E. et A.C. MORO (1990). Minori e Giustizia. Padova: Fondazione Zancan.

CIOLFI, T. et G. MILANA (1983). "Ipotesi di modello professionale dell'assistente sociale con riferimento alla più recente normativa sui servizi sociali ", Ministero dell'Interno: La formazione dell'assistente sociale. Rome.

COORDINAMENTO NAZIONALE DOCENTI DI SERVIZIO SOCIALE (1987). I/ servizio sociale come processo di aiuto. Milan: Franco Angeli.

DAL PRA PONTICELLI, M. (dir.) (1985). I modelli teorici del servizio sociale. Rome: Astrolabio.

DAL PRA PONTICELLI, M. (1987). Lineamenti di servizio sociale. Rome: Astrolabio.

DE LEONARDIS, O. (1990). II terzo escluso. Le istituzioni come vincoli e come risorse. Milan : Feltrinelli.

FERRARIO, F. et G. GOTTARDI (1985). " la relazione di aiuto: un possibile percorso di apprendimento », La Rivista di servizio sociale, $\mathrm{n}^{\circ} 3$.

FERRARIO, F. et G. GOTTARDI (1987). Territorio e servizio sociale: aspetti e problemi di un intervento. Milan: Unicopli.

GIRALDO, S. (1989). "Professionalità dell'assistente sociale nell'attuale fase di sviluppo dei servizi socio-sanitari : il contributo delle ricerche », Rassegna di servizio sociale, $\mathrm{n}^{\circ} 4$.

NEVE, E. (1991). "Dimensioni etiche nell'esercizio delle funzioni di aiuto, di controllo e di tutela ", VECCHIATO, T. et F. VILLA (dir.), La deontologia professionale nel servizio sociale. Milan: Vita e Pensiero.

NEVE, E. et M. NIERO (dir.). Il tirocinio. Modelli e strumenti dall'esperienza delle scuole di servizio sociale italiane. Milan: Franco Angeli.

NiERO, M. (1990). Rapporto sulle professioni sociali 1990: Scuole di servizio sociale di Venezia e Verona, Regione Veneto.

Olivetti ManOuKIAN, F. (1988). Stato dei servizi. Bologne: Il Mulino.

PITCH, T. (dir.) (1987). Diritto a rovescio. Naples: Edizioni Scientifiche Italiane. PITCH, T. (1989). Responsabilità limitate. Milan: Feltrinelli. 